\title{
Kursbuch 189
}

Lauter Lügen

Sabine am Orde Brief einer Leserin - Matthias Hansl Lüge, Bluff \& Co. • Gerhard Waldherr Deutschkunde - Armin Nassehi Po:Pu:Lis:Mus • Barbara Zehnpfennig Keine Lüge ohne Wahrheit • Fritz Breithaupt, Martin Kolmar Fakten oder Faketen? • Cord Riechelmann Können Tiere lügen? • Walter Schels Schönheitswettbewerb • Jan-Werner Müller Fake Volk? • Peter Felixberger Achtung: Wahrheit! • Ludger Heidbrink, Alexander Lorch Post-Truth-Management • André Kieserling Grenzschutz • Claudia Pichler Der Polt 
Kursbuch 189

Lauter Lügen 
Das Kursbuch erscheint viermal im Jahr.

Das Heft kostet einzeln $€ 19$,-

Das Jahresabo (4 Ausgaben) kostet €60,-

Im Internet: https://kursbuch.online

Sven Murmann Verlagsgesellschaft mbH

Miramar-Haus, Schopenstehl 15, 20095 Hamburg

Tel. 0 40/3980 83-0

V.i.S.d.P.: Peter Felixberger

(c) 2017 Sven Murmann Verlagsgesellschaft mbH, Hamburg

Alle Rechte für sämtliche Beiträge, auch der Übersetzung und der Wiedergabe durch Funk- und Fernsehsendungen und alle elektronischen Übermittlungen, vorbehalten.

ISBN 978-3-946514-45-9

ISSN 0023-5652

Herstellung und Gestaltung: Murmann Publishers GmbH, Hamburg

Druck: Steinmeier GmbH \& Co. KG, Deiningen

Printed in Germany

Zuschriften bitte per Mail an: kursbuch@kursbuch.online

Abonnenten-Service: abonnements@kursbuch.online

Pressevertrieb: PressUp GmbH, Wandsbeker Allee 1, 22041 Hamburg. www.pressup.de 